\title{
Effects of spatial filtering and lack of effects of visual imagery on pattern-contingent color aftereffects
}

\author{
HONG ZHOU and JAMES G. MAY \\ University of New Orleans, New Orleans, Louisiana
}

\begin{abstract}
Checkerboards contain fundamental two-dimensional Fourier components oriented $45^{\circ}$ from the edges of individual checks. Previous studies have shown that contingent color aftereffects following adaptation to chromatic checkerboard stimuli were associated with the fundamental components rather than the edges. In the present experiments, we measured contingent color aftereffects, using the method of constant stimuli, after subjects adapted to unfiltered checkerboards and checkerboards with the fundamental Fourier components removed. The adaptation stimuli were magenta (or green) squares and green (or magenta) diamonds; the test stimuli were vertical or oblique sine-wave gratings with different saturations. After adaptation to unfiltered checkerboards, aftereffects contingent on the fundamental components were obtained. In contrast, after adaptation to filtered stimuli, aftereffects of smaller magnitude were found to be aligned with the edges. The data support the previous findings of spatial-frequency-contingent color aftereffects with checkerboard adaptation stimuli and indicate that the aftereffects can be associated with edges if the fundamental components of adaptation stimuli are removed by spatial filtering. We reexamined the possibility of color aftereffects induced by imagery of checkerboards. Contrary to the previous reports, no significant aftereffects were obtained.
\end{abstract}

Kelly (1976) reported that two-dimensional Fourier analysis shows that the fundamental Fourier components of a checkerboard are oriented $45^{\circ}$ from the edges of individual checks, and none of the higher harmonics are aligned with the edges. In previous studies (Green, Corwin, \& Zemon, 1976; May \& Matteson, 1976), it was found that contingent color aftereffects (CAEs) were associated with the fundamental components of checkerboard stimuli rather than edges. Other reports showed that this effect occurs only with spatial frequencies above 1.0 cycles per degree (cpd; May, Agamy, \& Matteson, 1978; May, Matteson, Agamy, \& Castellanos, 1978). At low spatial frequencies, CAEs were found to be associated with the edges of individual checks. One possibility is that edge-sensitive mechanisms operate when large elements and low spatial frequencies are employed, but that Fouriersensitive mechanisms operate when small elements and high frequencies are used. Another possibility is that both edge and Fourier mechanisms operate at all element sizes and spatial frequencies, but that the typical contrast sensitivity profile renders the fundamental component of the checkerboard more visible than the components forming the edges when smaller checks are used. As the spatial frequency content of the checkerboard is lowered, observers may become less sensitive to the fundamental and

This research was supported by a grant from the Louisiana State University Board of Regents. Correspondence should be addressed to Hong Zhou, Department of Psychology, University of New Orleans, Lakefront, New Orleans, LA 70148. more sensitive to the higher harmonics aligned more closely with the check edges. In the present study, we used the method of constant stimuli to measure the contingent CAEs induced by the unfiltered checkerboards with a fundamental spatial frequency of $3.0 \mathrm{cpd}$. These stimuli had previously been shown to give rise to effects aligned with the fundamental components. In addition, we measured CAEs with the same checkerboards after the fundamental Fourier components had been removed by spatial filtering. Since the power of the higher harmonics is less than the fundamental, and since observers are less sensitive to these higher harmonics, we expected smaller magnitude CAEs induced by filtered adaptation stimuli.

A secondary aim of the present study was to reexamine the possibility of CAEs induced by imagery of checkerboards. Previous results regarding adaptation to imagery were conflicting. Finke and Schmidt $(1977,1978)$ reported that subjects asked to imagine vertical or horizontal bars on physically present homogeneous fields of red or green reported complementary aftereffects similar to those found by McCollough (1965). They also asked subjects to imagine green or red on physically present vertical or horizontal gratings, with similar results. Kunen and May (1980) reported that when subjects were asked to imagine orthogonal checkerboards on homogeneous fields of magenta and green, contingent CAEs were found to be aligned with the edges of the imagined checkerboards. Broerse and Crassini (1980), using different procedures, failed to find any support for the notion that imagined stimuli can substitute for real stimulation. Although it was originally felt (Kunen \& May, 1981) that this dis- 
crepancy might have derived from methodological differences, extensive attempts to replicate the positive findings have failed. ${ }^{1}$ In the present study, we employed a method of constant stimuli to once again investigate the possibility that contingent CAEs can be obtained with imagined checkerboards.

\section{METHOD}

\section{Subjects}

Ten experimentally naive student volunteers ( 5 males and 5 females) served as subjects. All had 20/20 acuity, uncorrected or corrected with lens, and all had normal color vision. Their ages ranged from 20 to 25 years.

\section{Stimuli and Apparatus}

All stimuli were generated with an image processing system (Data Translation DT 2851) and a microprocessor (Everex 1800) and were presented on a high resolution monitor (NEC DM 2000P). Adapting stimuli (Figure 1) included two pairs of checkerboards: (1) one with horizontal and vertical edges (squares) and the other with the edges oriented at $45^{\circ}$ (diamonds); (2) square and diamond checkerboards with the fundamental Fourier components removed by high-pass filtering. In addition, two blank chromatic fields (one magenta and one green) with the words "squares" or "diamonds" printed at the bottom of each were used. The test stimuli were ver- tical or oblique sine-wave gratings (Figure 1). Eleven test gratings of each orientation were used. The gratings varied in saturation from green $(-16)$ through white $(0)$ to magenta $(+16) .{ }^{2}$ The fundamental frequencies of checkerboards were equated with the test gratings $(3.0 \mathrm{cpd})$. The contrast of all stimuli was $70 \%$. The spaceaveraged luminance was $15.5 \mathrm{~cd} / \mathrm{m}^{2}$ for the checkerboards and $10.5 \mathrm{~cd} / \mathrm{m}^{2}$ for the test gratings. Field sizes subtended visual angles of $16^{\circ}$ for the adaptation stimuli and $8^{\circ}$ for the test gratings.

\section{Procedure}

In the pretest phase, the subjects were forced to decide if the color of the test gratings was either green or magenta. Each grating was presented 12 times in random order. Following the pretest, subjects adapted to alternate 5-sec presentations of each member of a pair for $12 \mathrm{~min}$. After this initial adaptation period, each of the 22 test gratings was presented once, and the subjects indicated whether they appeared green or magenta. This was followed by $2 \mathrm{~min}$ of adaptation. This adaptation-test sequence was repeated until 12 test trials had been accumulated for each test grating. The subjects were asked to participate in three experiments. First, they adapted to imaginary checkerboards using the blank chromatic stimuli. The subjects were instructed to imagine the squares on one color and diamonds on the other color during the adaptation period. In the second experiment, the subjects adapted to checkerboards with the fundamental components removed. In the third experiment, they adapted to the unfiltered stimuli. Adaptation color and pattern (or words) were reversed for half the subjects. At least 1 week elapsed between the subjects' participation in each experiment.
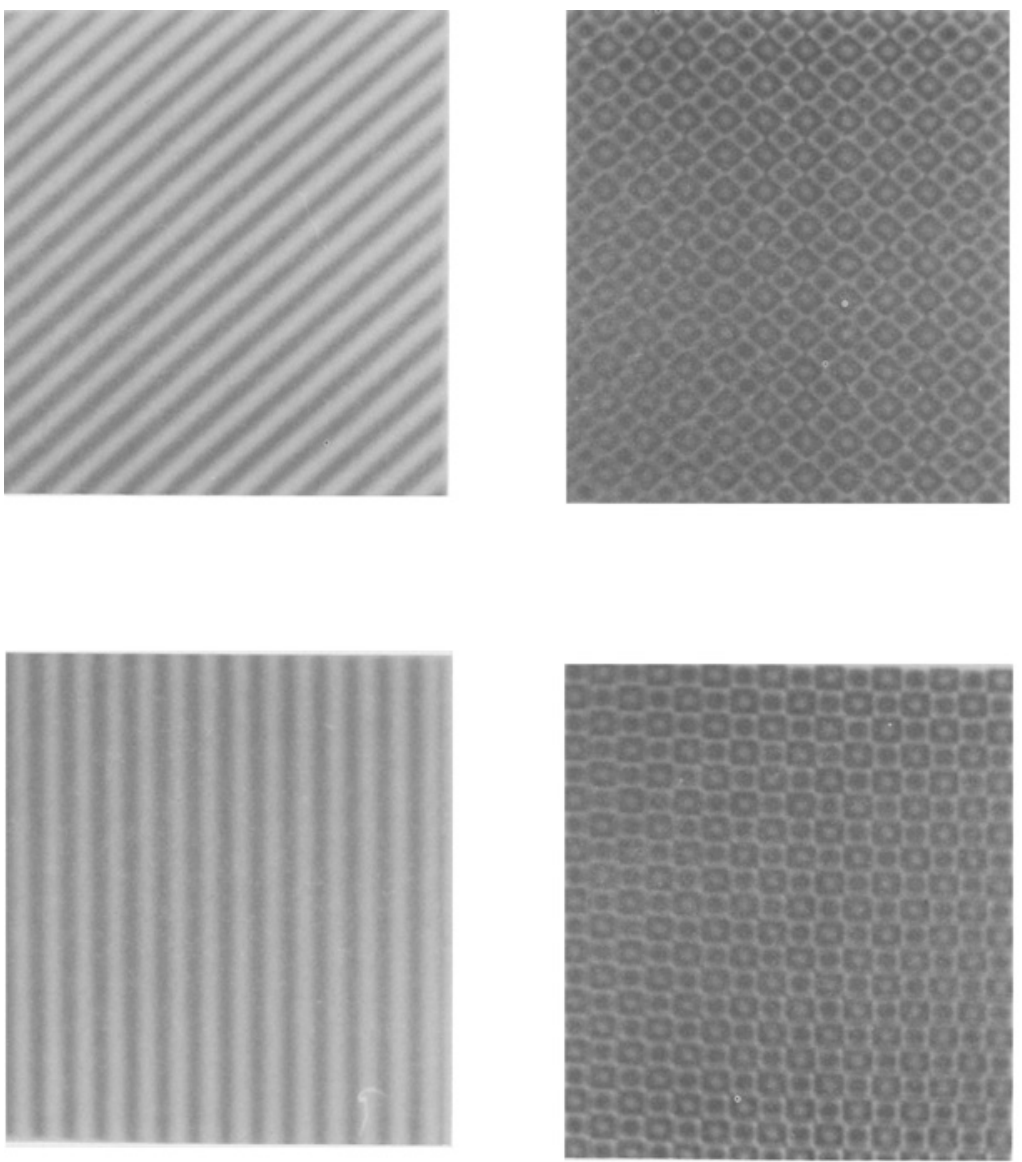
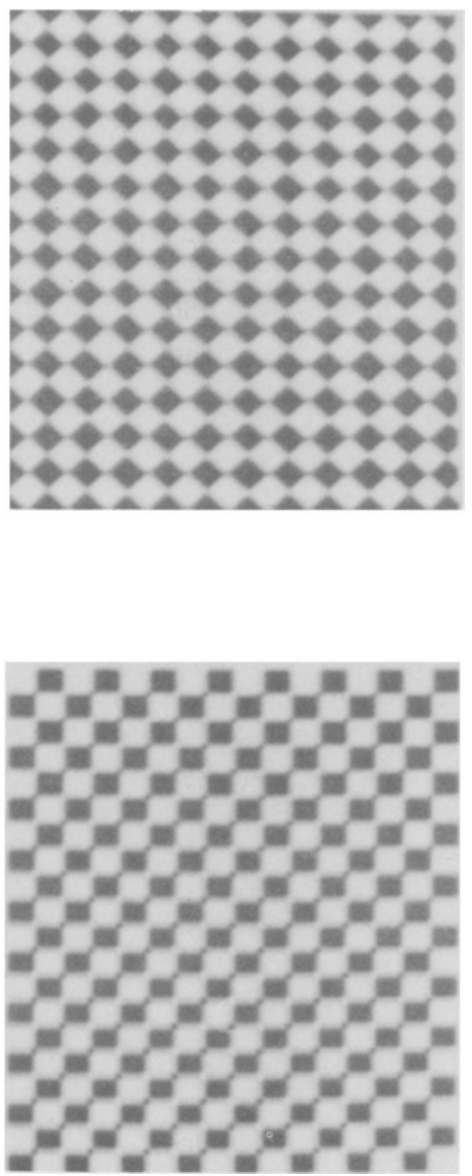

TESTING GRATINGS

Figure 1. Adaptation and test stimuli used in the experiment. 


\section{RESULTS}

For both the pretest and the posttest, the percentage of trials on which gratings were judged magenta or green for each level of saturation was obtained. These data were obtained for each subject under each adaptation condition, and each data set was fit by probit analysis. The means of each function provided the pre- and posttest match points for each subject. A typical pair of psychometric functions for oblique gratings after adaptation to unfiltered magenta squares and green diamonds are depicted in Figure 2. Before adaptation, the mean of the function is near zero. After adaptation, the mean of the function shifts toward magenta, indicating that the point at which oblique gratings are judged to be magenta or green equally often occurs when the gratings are tinted with magenta. Since the square checkerboard was magenta, this indicates that the aftereffects were associated with the fundamental Fourier component. Shifts in the opposite direction were observed with vertical gratings after the same adaptation. The sign of the data for the group serving in one color/ pattern condition was reversed so that the direction of change was the same as that in the other color/pattern condition. This coding resulted in positive change for vertical gratings and negative change for oblique gratings when the aftereffects were associated with the fundamental Fou-

\section{PSYCHOMETRIC FUNCTION}

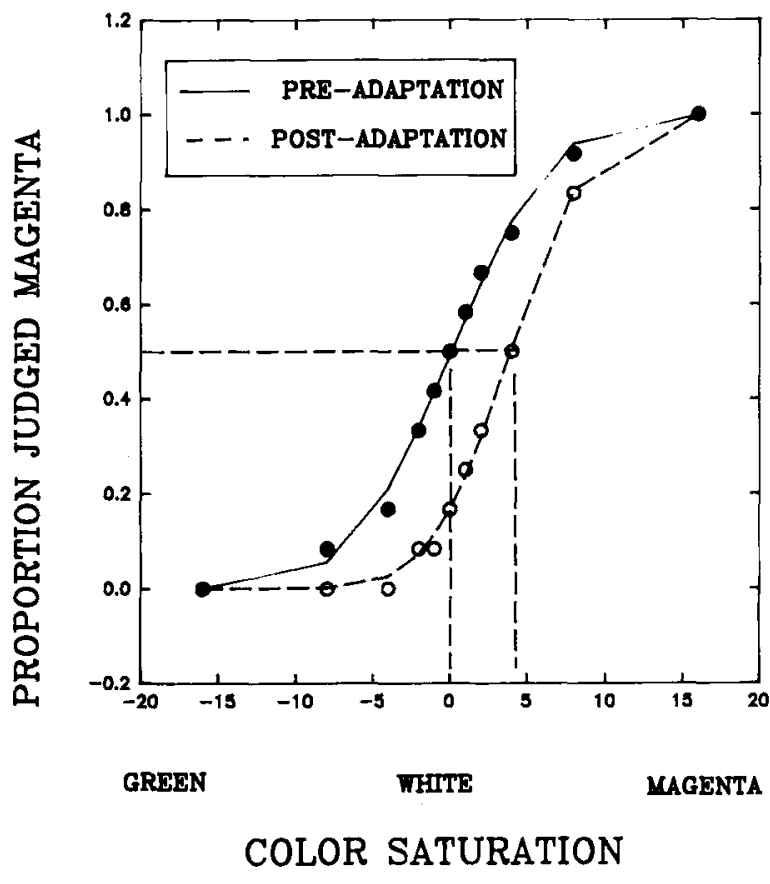

Figure 2. A typical pair of psychometric functions obtained with an oblique grating before (closed circles) and after (open circles) adaptation to magenta squares and green diamonds. The magnitude of the aftereffect is indicated by the difference in the dotted vertical lines.

\section{UNFILTERED CHECKERBOARDS}

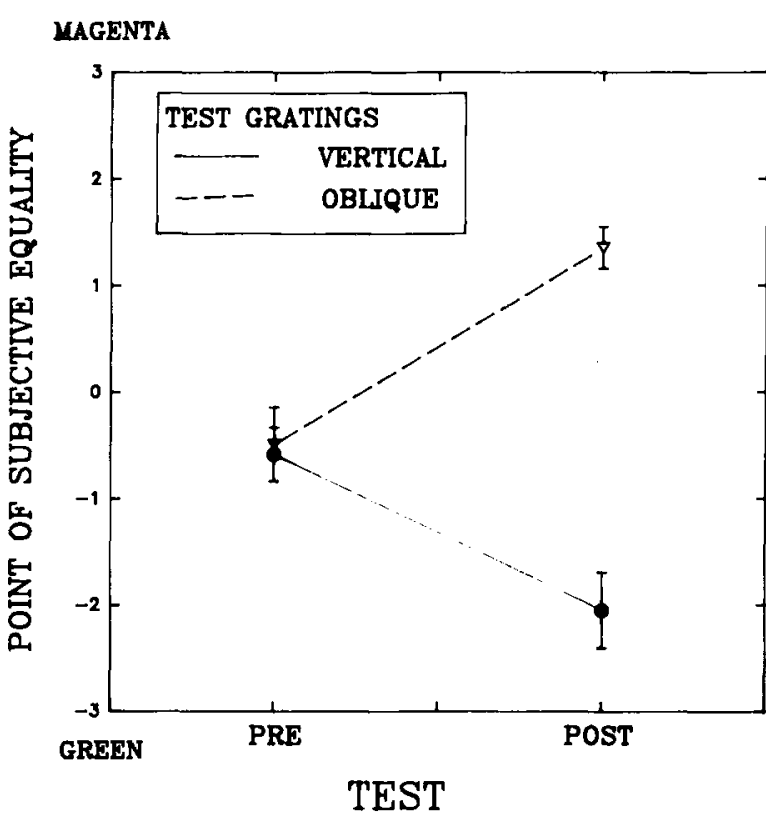

Figure 3. Mean match points for pre- and posttest measures with oblique and vertical gratings when unfiltered checkerboards were used during adaptation. Afterefiects associated with the fundamental Fourier components are indicated by positive shifts for oblique gratings and negative shifts for vertical gratings.

rier components. Negative changes for vertical gratings and positive changes for oblique gratings indicated that the aftereffects were associated with the edges. For the unfiltered checkerboards (Figure 3), the mean pretest scores for vertical and oblique gratings were near zero, but after adaptation, the mean posttest scores were more positive for vertical gratings and more negative for oblique gratings, indicating that the aftereffects were aligned with the fundamental Fourier components. For filtered checkerboards (Figure 4), the reverse was true. Mean pretest scores for both gratings were near zero, but the posttest scores for vertical gratings became more negative and the posttest scores for oblique gratings became more positive, indicating that the aftereffects were aligned with the edges. For the imagery condition, very little pre-post difference was noted for either grating. The data for each adaptation condition were submitted to separate two-way mixed analyses of variance (orientation $x$ adaptation conditions). Significant interactions were obtained for unfiltered checkerboards $[F(1,9)=39.86, p<.0001]$ and for filtered checkerboards $[F(1,9)=12.71, p<.0061]$. No significant main effects or interactions were obtained for the imagery condition. With the unfiltered checkerboards, the CAEs were aligned with the fundamental frequencies of the adaptation pattern, whereas with the filtered checkerboards, which were devoid of the fundamental Fourier components, the CAEs were contingent on the edges. The CAE magnitude (pretest score - posttest score) was greater for unfiltered checkerboards than for filtered 


\section{FILTERED CHECKERBOARDS}

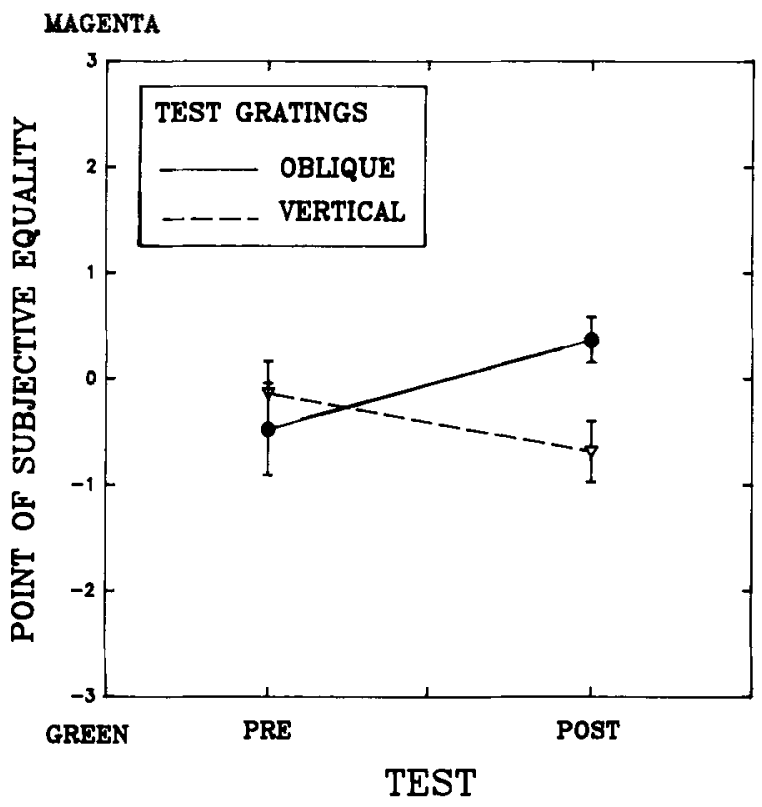

Figure 4. Mean match points for pre- and posttest measures with oblique and vertical gratings when filtered checkerboards were used during adaptation. Aftereffects associated with the edges are indicated by negative shifts for oblique gratings and positive shifts for vertical gratings.

checkerboards. Tests subsequent to analysis of variance (Newman-Keuls) indicated that the pre- and posttest means were significantly different for both gratings after adaptation to unfiltered checkerboards $(p<.003)$, but significant pre-post differences were obtained only with the oblique grating after adaptation to filtered checkerboards $(p<.02)$.

\section{DISCUSSION}

The present results agree with previous results obtained with the use of unfiltered checkerboard adapting stimuli, in that contingent CAEs were found to be aligned with the fundamental Fourier components of the stimuli rather than the edges. However, when the fundamental Fourier components were removed from checkerboards with highpass filtering, CAEs were found to be aligned with the edges of the checks. This suggests that mechanisms that mediate the perception of edges and fundamental Fourier components were both adapted when unfiltered checkerboards were used, but since the two adaptational effects were set in opposition, only the stronger effects were observable. The original notion was that the checkerboard adaptation paradigm would provide a critical test concerning whether the mechanisms in the visual system were spatial frequency analyzers or edge detectors. It is clear that there exist mechanisms sensitive to spatial frequency components, but it is also clear that filtered checkerboards give rise to edge effects. The question that remains is whether there are edge detectors apart from spatial frequency analyzers. This question remains unanswered by the present data because, although the higher harmonics of the checkerboard are not aligned with the check edges, they are oriented arbitrarily close to those meridians. The orientational tuning of spatial frequency channels is broad enough to allow spatial frequency channels aligned with the edges to be adapted by the higher harmonics in the checkerboard. Future experiments that assess CAE magnitude with test stimuli varying more widely in orientation and spatial frequency will shed light on these possibilities. It is interesting to note that Webster, Day, Gillies, and Crassini (1992) reported that CAEs were produced on the adapted checkerboard and on sine-wave gratings aligned with the fundamental and third harmonics of the checkerboard spectrum when a 4-cpd checkerboard was adapted; adaptation of a coarser checkerboard $(0.80 \mathrm{cpd})$ produced CAEs aligned with both the edges and the harmonic frequencies. They concluded that spatial frequency mechanisms are operating at both low and high spatial frequencies and that an edge mechanism is operative at lower frequencies. Likewise, it is probably the broad tuning spatial frequency channels, adapted by the higher harmonics in the filtered checkerboard, that give rise to the edge effects found in our data.

The results of the present study offer no support for the notion that imagery gives rise to adaptation of these sensory mechanisms. This finding agrees with those of Broerse and Crassini $(1980,1981)$ and contradicts reports by Finke and Schmidt (1977), Kunen and May (1980), and Kaufman, May, and Kunen (1981). In the latter two studies, subjects turned a knob to null out differences in color between vertical and oblique gratings. The experimenter selected the starting point for each trial. In addition, the strong adaptational effects gave rise to a much greater interval of uncertainty for color matches. These conditions may have increased the possibility of experimenter and/or subject biases. In the present study, stimuli were under computer control and the experimenter was less involved in the pre- and posttesting. Allan, Siegel, Toppan, and Lockhead (1991) reported that the method of constant stimuli is less susceptible to experimenter/subject bias. Although imagery may well employ the same neural mechanisms as actual perception, this must be questioned until reliable support can be demonstrated.

\section{REFERENCES}

Allan, L. G., Siegel, S., Toppan, P., \& Lockhead, G. R. (1991). Assessment of the McCollough effect by a shift in psychometric function. Bulletin of the Psychonomic Society, 29, 21-24.

Broerse, I., \& Crassini, B. (1980). The influence of imagery ability on color aftereffects produced by physically present and imagined induction stimuli. Perception \& Psychophysics, 28, 560-568.

Broerse, J., \& Crassini, B. (1981). Misinterpretations of imageryinduced McCollough effects: A reply to Finke. Perception \& Psychophysics, 30, 96-98.

FINKE, R. A., \& SCHMIDT, M. J. (1977). Orientation-specific color aftereffects following imagination. Journal of Experimental Psychology: Human Perception \& Performance, 3, 599-606. 
FINKE, R. A., \& SCHMIDT, M. J. (1978). The quantitative measure of pattern representation in images using orientation-specific color aftereffects. Perception \& Psychophysics, 23, 515-520.

Green, M., Corwin, T. R., Z Zemon, V. (1976). A comparison of Fourier analysis and feature analysis in pattern-specific color aftereffects. Science, 192, 147-148.

Kaufman, J. H., MaY, J. G., \& Kunen, S. (1981). Interocular transfer of orientation-contingent color aftereffects with external and internal adaptation. Perception \& Psychophysics, 30, 547-551.

Kelly, D. H. (1976). Pattern detection and the two-dimensional Fou rier transform: Flickering checkerboards and chromatic mechanisms. Vision Research, 16, 277-289.

Kunen, S., \& MAY, J. G. (1980). Spatial frequency content of visual imagery. Perception \& Psychophysics, 28, 555-559.

KUNEN, S., \& MAY, J. G. (1981). Imagery-induced McCollough effects: Real or imagined? Perception \& Psychophysics, 30, 99-100.

May, J. G., AGamy, G., \& Matteson, H. H. (1978). The range of spatial frequency contingent color aftereffects. Vision Research, 18 , 917-921.

MAY, J. G., \& MATteson, H. H. (1976). Spatial frequency contingent color aftereffects. Science, 192, 145-147.

May, J. G., Matteson, H. H., Agamy, G., \& Castellanos, P. (1978). The effects of differential adaptation on spatial frequencycontingent color aftereffects. Perception \& Psychophysics, 23, 409-412.

McCollough, C. (1965). Color adaptation of edge detectors in the human visual system. Science, 149, 1115-1116.
Webster, W. R., Day, R. H., Gillies, O., \& Crassini, B. (1992) Spatial-frequency-contingent color aftereffects: Adaptation with twodimensional stimulus patterns. Perception \& Psychophysics, 51, 66-78.

\section{NOTES}

1. With an apparatus and procedures quite similar to those employed by Kunen and May (1980), the second author carried out two extensive experiments in collaboration with $\mathrm{J}$. Broerse and B. Crassini in the Department of Psychology at the University of Queensland. Reliable pattern-contingent CAEs were obtained with actual stimuli, but neither experiment revealed aftereffects following imagery conditions

2 . Variation in saturation was achieved by passing the grating images through lookup tables that specified the luminance of the red, green, and blue guns of the color monitor. When all three guns were set to the same levels, the gratings appeared white. To the degree that the red and blue guns were incremented and the green gun was decremented, the gratings appeared to be a more saturated magenta. As red and blue were decremented while green was incremented, the gratings appeared to be a more saturated green.

(Manuscript received December 9, 1991; revision accepted for publication July 24, 1992.) 